\title{
Topologically non-trivial field configurations - interplay of vortices and Dirac eigenmodes
}

\author{
Stefan Solbrig ${ }^{* a}$, Jochen Gattnar $^{b}$, Christof Gattringer $^{c}$, Kurt Langfeld ${ }^{b}$, \\ Hugo Reinhardt ${ }^{b} ;$ Andreas Schäfer ${ }^{a}$, Torsten Tok ${ }^{b}$ \\ ${ }^{a}$ University of Regensburg, Germany \\ ${ }^{b}$ University of Tübingen, Germany \\ ${ }^{c}$ University of Graz, Austria \\ E-mail: stefan.solbrig@physik.uni-r.de
}

\begin{abstract}
Gluon field configurations with non-trivial topology like instantons, magnetic monopoles and center vortices play a crucial role in QCD and, in particular, for the spontaneous breaking of chiral symmetry. Moreover, center vortices are strongly correlated with confinement. We present evidence, that there is a deep connection between the topology of gauge fields and center vortices. We use the chirally improved lattice Dirac operator to compute eigenvectors and eigenvalues of various lattice gauge field configurations. Removing vortices from thermalized configurations also removes the topological content of the gauge field. As a consistency check, we apply random changes to the raw configurations.
\end{abstract}

XXIIIrd International Symposium on Lattice Field Theory

25-30 July 2005

Trinity College, Dublin, Ireland

\footnotetext{
* Speaker.

${ }^{\dagger}$ supported by BMBF and DFG

† supported in part by DFG Re 856/5-1
} 


\section{Introduction}

It has long been known that the low lying eigenmodes of the Dirac operator are a good tool to detect infrared, localized structures in lattice gauge fields. In addition, the spectrum of the Dirac operator allows to detect chiral symmetry breaking via the Banks-Casher relation [1]. The breaking of chiral symmetry is usually attributed to instanton-like structures, while center vortices are considered to be responsible for confinement. However, both structures, instantons and vortices, give rise to zero modes of the Dirac operator [2]. This is in accord with the Atiyah-Singer-Index theorem [3], since both types of configurations carry topological charge. In the case of center vortices topological charge is realized by vortex intersection [4, 5] and writhing [5].

We will use the properties of Dirac eigenvalues and eigenvectors to investigate the relation between vortices, chiral symmetry and topology [6]. We used quenched $S U(2)$ configurations. All the results presented here are for the Chirally Improved Dirac operator [7, 8].

The first step is to identify vortices on the lattice. This is usually done after a suitable gauge fixing. A common choice is the maximal center gauge. In our case, the quantity

$$
R=\frac{1}{4 V} \sum_{x \in \text { lattice }} \sum_{\mu}\left[\frac{1}{2} \operatorname{tr}\left(U_{\mu}^{g}(x)\right)\right]\left[\overline{\frac{1}{2} \operatorname{tr}\left(U_{\mu}^{g}(x)\right)}\right]
$$

is maximized by applying gauge transformations $g$. In the above equation, $V$ is the number of lattice sites and $U_{\mu}^{g}(x)$ is the link variable after a gauge transformation $g$.

For $S U(2)$, the center projected link variable $Z_{\mu}(x)$ is defined as:

$$
Z_{\mu}(x)=\operatorname{sign} \operatorname{Re} \operatorname{tr}\left(U_{\mu}(x)\right) .
$$

\section{Vortex Removal}

If we want to understand how vortices influence the spectrum of the Dirac operator, the following idea might come to your mind: Perform gauge fixing and a subsequent center projection as described above, in order to produce "vortex only" configurations. Then compute the eigenvalues and eigenvectors for these configurations directly. However, it turns out that this approach is too naive. The center projected configurations consist entirely of links that are either $+\mathbf{1}$ or $-\mathbf{1}$. In this sense, they are maximally discontinuous and not suitable for the analysis with our Dirac operator. Another approach has to be followed.

The method of choice is the removal of center vortices, as introduced in [9]. The new configurations, called "vortex removed configurations," are given by

$$
U_{\mu}^{\mathrm{rem} .}(x)=U_{\mu}(x) Z_{\mu}^{\dagger}(x)
$$

for every lattice site $x$. They are void of center vortices in the sense that center projection of a vortex removed configuration yields a trivial gauge configuration.

Let us first look at the plots in Figure 1. On the left hand side, we show a superposition of ten spectra for the original ("raw") configurations. The eigenvalues are close to the GinspargWilson circle, just as one would expect for the Chirally Improved Dirac operator. The eigenvalues extend all the way down to real eigenvalues, which shows that the underlying configurations are in 


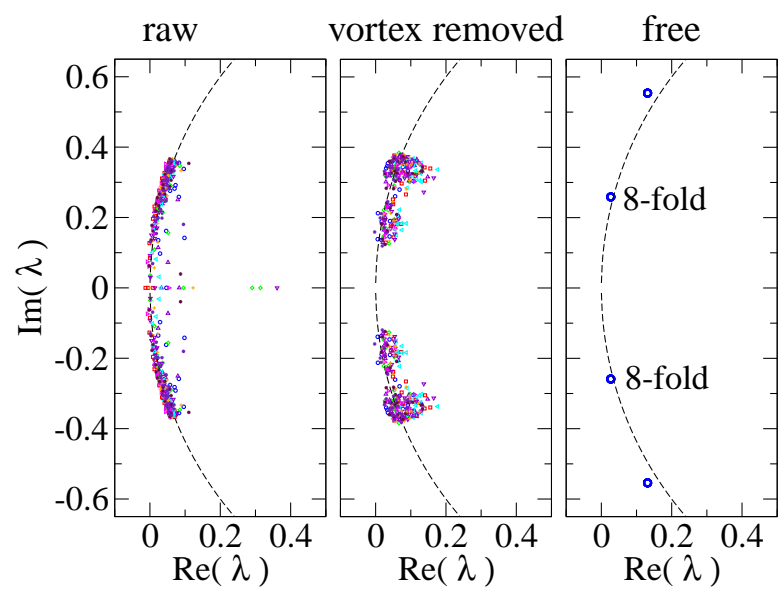

Figure 1: Dirac spectra for the Chirally Improved Dirac operator for various gauge field configurations. Each plot contains the superimposed spectra for 10 gauge field configurations. All spectra have been computed for anti-periodic fermionic boundary conditions in the time direction.

the chirally broken phase. There are also many real eigenvalues, i.e., there are Dirac zero modes connected with the raw configurations. This shows that many raw configurations are topologically non-trivial.

The spectra of the vortex removed configurations show a completely different picture: There are no more real eigenvalues and thus no more topological modes. In addition, a large gap opened around the origin. The Banks-Casher relation [1] tells us, that we are now in the chirally restored phase. Since the eigenvalues stay close to the Ginsparg-Wilson circle, we know that our Dirac operator works well for vortex removed configurations.

A prominent feature is, that the eigenvalues for vortex removed configurations form clusters in the complex plane. If one looks closely, one sees that there are eight eigenvalues per configuration in the lowest cluster. We would like to point out that the degeneracy of the lowest eigenvalue for a free field is also eight (anti-periodic temporal b.c.). The spectrum of the free field is shown in the right hand side plot of Figure 1.

\section{Distribution of Eigenvalues}

We want to probe the clustering of eigenvalues, as mentioned in the previous section. In particular, we want to know if there is a connection between the number of eigenvalues in a cluster and the degeneracy of the eigenvalues for a trivial field. For this purpose we modify the boundary condition of the fermion field in the time direction: When the fermion fields wind once around the lattice in the time direction, they can acquire a phase. All other directions have periodic boundary conditions. Thus the eigenvectors $\vec{v}$ obey

$$
\vec{v}(x+L \hat{\imath})=\vec{v}(x), \quad \hat{\imath}=\widehat{1}, \widehat{2}, \widehat{3}, \quad \vec{v}(x+L \widehat{4})=e^{i 2 \pi \zeta} \vec{v}(x),
$$

where $L$ is the size of the lattice, $\hat{\imath}$ are the unit vectors in the spatial directions and $\widehat{4}$ is the unit vector in the time direction. 

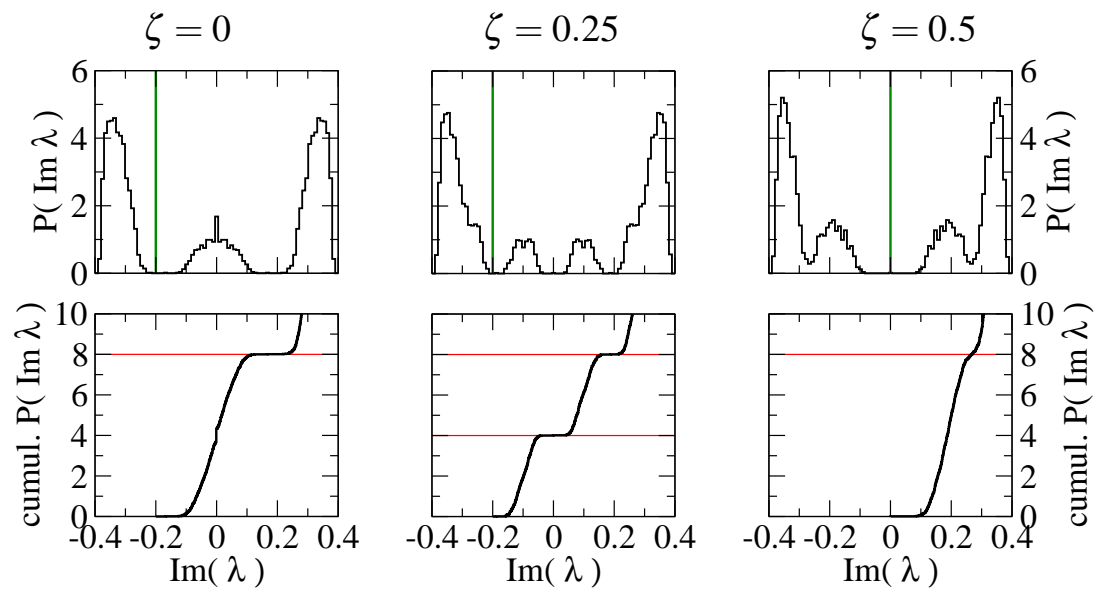

Figure 2: Histograms and cumulative histograms for the imaginary part of the Dirac spectra.

The important fact is, that the degeneracy of the eigenvalues for a free field depends on the parameter $\zeta$. It is 8 in the case of $\zeta=0, \zeta=1 / 2$ and it is 4 in the case of $\zeta=1 / 4$.

Figure 2 shows histograms and cumulative histograms of Dirac spectra for vortex removed configurations. We plot the density of eigenvalues as a function of $\operatorname{Im} \lambda$. The upper row shows regular, normalized histograms. The lower row shows cumulative histograms, normalized by the number of configurations used. The vertical line marks the starting point for the cumulative histograms. Clearly, we see that the lumpy structure of the spectrum as seen in Figure 1 is reflected by the peaks in the regular histograms. These peaks then show up as plateaus and kinks in the cumulative histograms. The horizontal lines mark the plateaus and kinks. We see that the position of plateaus and kinks of the cumulative histograms nicely matches the degeneracy of the free Dirac operator for a given value of $\zeta$. This is an indication that the vortex removed configurations are almost trivial configurations.

\section{The Effects of Random Changes}

The removal of vortices is quite a drastic change to the configuration. In our case, almost half of the links were changed. However, these changes occur in a highly non-trivial manner. Yet, one could criticize the vortex removal: Suppose the underlying topological structures of the gauge field are sensitive to arbitrary small changes of the configuration. This would mean that vortex removal cannot make a statement about the nature of the topological structures. To invalidate this critique, we apply random changes to the configuration. If the topological structures survive this test, we conclude that the vortices are strongly correlated with the relevant topological structures.

One question remains: How many links do we have to change at random in order to perform a sensible test? The number of links, that are changed to remove the vortices, is surely not a good quantity. Note that the gauge fixing in Eq. (1.1) does not fix the gauge completely: There is a remaining $\mathbb{Z}_{2}$ gauge freedom that can be used to vary the number of negative links. Thus, instead of using the total number of random changes, we use the average plaquette $\left\langle U_{p}\right\rangle$ as a measure to determine how many links we should change randomly. Table 1 gives the overview of the situation. 


\begin{tabular}{l|r|r|r|} 
& links changed & plaquettes changed & $\left\langle U_{p}\right\rangle$ \\
\hline original (raw) configurations & none & none & 0.651 \\
\hline vortex removed configurations & $50.0 \%$ & $3.3 \%$ & 0.621 \\
\hline randomly changed configurations & $1.2 \%$ & $4.6 \%$ & 0.590 \\
& $6.0 \%$ & $20.1 \%$ & 0.389 \\
& $24.1 \%$ & $46.5 \%$ & 0.046
\end{tabular}

Table 1: Comparison of the number of changed links, changed plaquettes and the change of the average plaquette for vortex removed and randomly changed configurations.

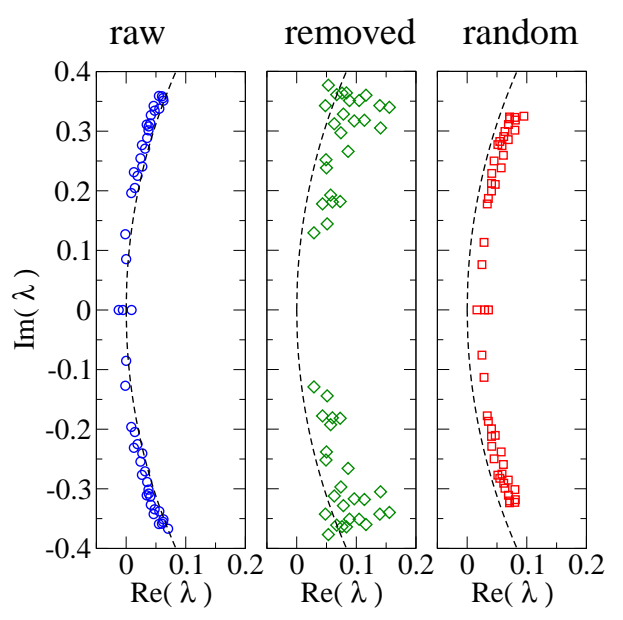

Figure 3: Dirac spectrum of a single configuration. It is shown for the unchanged, (raw) configuration, the same configuration with the vortices removed, and with random changes applied to the raw configuration.

For our randomly changed configurations, we changed 1000 links, which corresponds to changing $1.2 \%$ of the links on a $12^{4}$ lattice.

Figure 3 shows the effect for a single configuration. As discussed above, the vortex removed spectrum looks completely different. In contrast, the spectrum for the randomly changed configuration looks essentially like the original (raw) spectrum. In particular, the number of zero modes did not change by the random changes. In Figure 4 we show the distribution of the topological charge for 100 configurations before and after random changes. We see that the overall shape of the distribution did not change.

\section{Conclusions and Outlook}

We can conclude that removal of the vortices produces configurations that resemble free fields. These vortex removed fields are void of topological structures and chiral symmetry is restored. Applying random changes to the original configuration shows that the removal of vortices is indeed a sensible method to probe the connection between vortex-like structures and topology. For $\mathbb{Z}_{2}$ projected configurations the situation is different. In the case of $\mathbb{Z}_{2}$ configurations that have been derived from thermalized configurations, the fermionic analysis does not yield sensible results. 

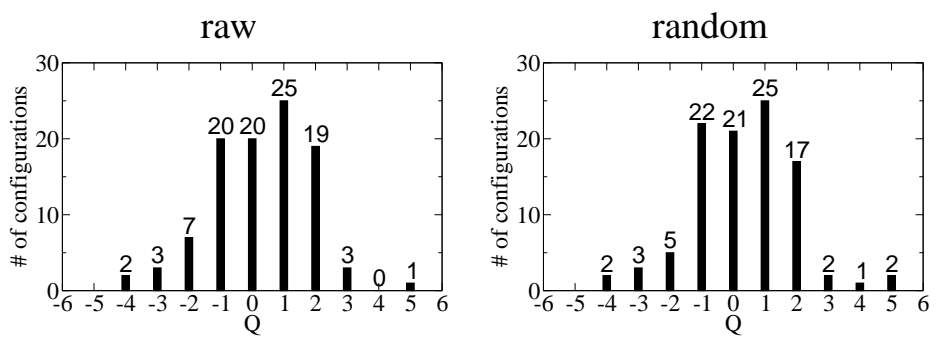

Figure 4: This figure shows the distribution of the topological charge $Q$ for raw and randomly changed configurations. Vortex removed configurations always have $Q=0$.

However, in the case of hand constructed vortices, analytic results are reproduced. Especially, the low-lying, non-zero modes of the Dirac operator do feel the presence of vortices, even for center projected configurations. For a detailed discussion of hand constructed configurations see [6].

\section{References}

[1] T. Banks and A. Casher, Chiral symmetry breaking in confining theories, Nucl. Phys. B169 (1980) 103.

[2] H. Reinhardt, O. Schroeder, T. Tok, and V. C. Zhukovsky, Quark zero modes in intersecting center vortex gauge fields, Phys. Rev. D66 (2002) 085004, hep-th/0203012.

[3] M. F. Atiyah and I. M. Singer, The index of elliptic operators. 5, Annals Math. 93 (1971) 139-149.

[4] M. Engelhardt and H. Reinhardt, Center projection vortices in continuum Yang-Mills theory, Nucl. Phys. B567 (2000) 249, hep-th/9907139.

[5] H. Reinhardt, Topology of center vortices, Nucl. Phys. B628 (2002) 133-166, hep-th/0112215.

[6] J. Gattnar et al., Center vortices and Dirac eigenmodes in SU(2) lattice gauge theory, Nucl. Phys. B716 (2005) 105-127, hep-lat/0412032.

[7] C. Gattringer, A new approach to Ginsparg-Wilson fermions, Phys. Rev. D63 (2001) 114501, hep-lat/0003005.

[8] C. Gattringer, I. Hip, and C. B. Lang, Approximate Ginsparg-Wilson fermions: A first test, Nucl. Phys. B597 (2001) 451-474, hep-lat/0007042.

[9] P. de Forcrand and M. D'Elia, On the relevance of center vortices to QCD, Phys. Rev. Lett. 82 (1999) 4582-4585, hep-lat/9901020. 